\title{
Methods for calculating PNECs using species sensitivity distribution (SSD) with various hypothesis on the way to handle ecotoxicity data
}

\author{
P. Ciffroy \\ EDF Research and Development, Département Laboratoire National \\ d'Hydraulique et Environnement, Chatou, France
}

\section{Abstract}

Species sensitivity distribution (SSD) methodology is currently used to determine the Predicted No Effect Concentration (PNEC) of a substance, considering chronic ecotoxicological tests on three taxonomic groups: algae, invertebrates and vertebrates (aquatic environment). However, SSD methodology raises some practical questions due to the multiple possibilities of handling the available data. In particular the following points are often discussed: (1) how to take into account the intra-species variation; (2) how to handle heterogeneous repartition of available data among the taxonomic groups; (3) could we use the information contained in acute data, and how, in the construction of SSDs; (4) when only a limited number of data is available, how is it possible to build relevant SSDs. Different approaches have been tested in answer to each of these questions: For questions (1) and (2): different methods were compared, each of them being characterized by a different way of taking into account intra-species variation and proportions of taxonomic groups (vertebrates, invertebrates and algae), as well as by the actual method of calculation of the HC5 (Hazardous Concentration) and its confidence interval. These different methods were compared for 15 substances using NOEC data available in the literature and the uncertainty associated to the data treatment was quantified. For question (3): an "Acute-to-Chronic Transformation" (ACT) function was derived and a methodology for the incorporation of acute data in the construction of SSDs was tested. This ACT method was tested on 11 substances: for each substance, the actual chronic SSD was compared to the predicted SSD derived from the incorporation of transformed acute data. For question (4): Bayesian methods were tested to build SSDs from limited datasets and the relevance of such methods was discussed. Various approaches are suggested here in answer to each of these three questions. They have been combined in a comprehensive overview to define alternative strategies according to the availability of ecotoxicological data.

Keywords: risk assessment, species sensitivity distribution, PNEC, uncertainty. 


\section{Introduction}

The so-called "Species Sensitivity Distribution" (SSD) methodology has been used since 1996 in the European Community [1] in order to determine the "Predicted No Effect Concentration" (PNEC) of a substance, where chronic ecotoxological tests carried out on the substance are numerous enough (a minimum of fifteen or so). For the aquatic environment with which we are concerned, the three taxonomic groups, algae, invertebrates and vertebrates, need to be represented. This approach is based on the hypothesis that the species for which results of ecotoxicological tests are known are representative, in terms of sensitivity, of the totality of the species in the environment (e.g. see Aldenberg and Slob [2]). A likely distribution of species sensitivity is then estimated from these results, which enables a concentration that is assumed to protect a given percentage of the species in the environment to be calculated. The agreed European concentration is $\mathrm{HC5} 50 \%$, the hazardous concentration protecting $95 \%$ of species with $50 \%$ confidence.

The SSD approach raises a number of questions (e.g. see Forbes and Calow [3]). From a practical point of view, irrespective of validation and criteria used for selecting the results of ecotoxicological tests used in SSD (criteria which we shall not discuss here), we will concentrate on the following questions:

- When the chronic ecotoxicological results are numerous enough:

1. Should intra-species variation be taken into account? And if so, how?

2. How do we take into account the fact that the number of available data varies from one category of species to another (between vertebrates, invertebrates and algae)?

- When chronic ecotoxicological results are limited, but acute ecotoxicological tests are numerous enough:

3. Can acute toxicity data for a substance give us information about the chronic toxicity of the same substance and be used for building chronic SSD?

- When ecotoxicological tests are scarce (typically less than 10):

4. is it possible to build relevant SSDs through techniques adapted for the treatment of small samples like Bayesian methods?

Various approaches are suggested here in answer to each of these questions. They have been combined in a comprehensive overview to define alternative strategies according to the availability of ecotoxicological data.

\subsection{Strategies for large chronic datasets}

\subsubsection{Materials and methods}

(1) Taking into account intra-species variation

In the method commonly used (European Commission [1]) for building SSDs in the case of large datasets of chronic ecotoxicological tests, the various test results that may exist for the same species (e.g. for daphnia) are averaged; as a result, a 
single value per species (the geometric mean) is used to determine the SSD. Because of differences in experimental conditions, strains of species, stages of development, etc, the intra-species variation between test results is frequently observed to be very high, and is sometimes equivalent to the inter-species variation. In the current methodology, this source of variability (the intra-species variation) is ignored. While it is not legitimate to ignore a priori intra-species variation by calculating a mean of the data, neither is it satisfactory to consider the set of data as it stands without taking into account the label "species", since one species is frequently much more abundant than the others with respect to the number of data (especially daphnia).

In order to study the effect of taking intra-species variation into account or not, Duboudin et al (2004) [4] compared three approaches that stem directly from the above discussion:

(1.1) The entire set of data available is treated as if they all belonged to different species. Intra-species variation is taken into account in the same way as inter-species variation, but there is a risk of giving too much importance to one species if the number of data we have for one species is high.

(1.2) As is done in the usual method, a geometric mean of data for each species is first calculated. In this case, intra-species variation is ignored.

(1.3) All the data are used, but each piece of data is weighted in order to give each species the same weight within the SSD. Intraspecies variation is taken into account, and no species is given more importance than any other.

(2) Taking into account the relative distribution of data among the three taxonomic groups: vertebrates, invertebrates and algae

For the determination of an aquatic PNEC, the SSD method is based on breaking down species into three taxonomic groups: vertebrates, invertebrates and algae. In most cases, it is necessary to incorporate these three taxonomic groups into the same SSD, which then raises the question of the proportions of data taken into account for each group.

Most research uses the data available in the literature and implicitly assumes that its distribution among the three species previously described is representative of the proportions existing in the environment. However, as a general rule, the representativity of laboratory species as compared to species in the environment is not guaranteed (Forbes and Calow [3]), because lab species are chosen because they are easy to breed and they are not the result of a random sample from among all the species. Thus, letting literature references decide which proportions of data are used to construct an SSD does not seem to be very satisfactory, since these proportions vary from one toxicant to another.

Duboudin et al [4] compared three approaches to study how much effect the distribution of data among taxonomic groups has:

(2.1) As is done in the usual method, the proportions of data given in the literature are retained. Ultimately, no distinction is made between the three taxonomic groups. 
(2.2) The data is balanced, in such a way that the three taxonomic groups are equally weighted in the SSD.

(2.3) The data is weighted in such a way as to respect the proportions proposed by Forbes and Calow [3]: 10\%, 26\% and 64\% for vertebrates, invertebrates and algae respectively.

Table 1: Statistical characteristics of results associated with 63 methods of calculation of HC5 toxicant by toxicant.

\begin{tabular}{|l|c|c|c|c|c|c|}
\hline \multirow{2}{*}{ Toxicant : } & \multicolumn{3}{|c|}{ Deviation to the mean } & \multicolumn{2}{c|}{ Amplitude } \\
\cline { 2 - 7 } & min & $\begin{array}{c}\text { 25th } \\
\text { percentile } \\
\text { (q25) }\end{array}$ & $\begin{array}{c}\text { 75th } \\
\text { percentile } \\
\text { (q75) }\end{array}$ & max & q75-q25 & max-min \\
\hline atrazine & -0.41 & -0.16 & 0.13 & 0.48 & 0.29 & 0.89 \\
\hline boric acid & -0.58 & -0.28 & 0.27 & 0.61 & 0.56 & 1.19 \\
\hline $\begin{array}{l}\text { butylbenzyl } \\
\text { phthalate }\end{array}$ & -0.11 & -0.05 & 0.04 & 0.19 & 0.09 & 0.30 \\
\hline cadmium & -0.21 & -0.07 & 0.09 & 0.19 & 0.15 & 0.41 \\
\hline chromium & -0.27 & -0.09 & 0.11 & 0.37 & 0.21 & 0.65 \\
\hline copper & -0.31 & -0.18 & 0.16 & 0.30 & 0.33 & 0.61 \\
\hline dibutyl phthalate & -0.21 & -0.13 & 0.03 & 0.42 & 0.17 & 0.63 \\
\hline dodmac & -0.35 & -0.15 & 0.13 & 0.30 & 0.28 & 0.64 \\
\hline lead & -0.24 & -0.10 & 0.09 & 0.29 & 0.20 & 0.53 \\
\hline lindane & -0.53 & -0.37 & 0.31 & 0.54 & 0.68 & 1.07 \\
\hline mercury & -0.54 & -0.21 & 0.31 & 0.59 & 0.52 & 1.13 \\
\hline nickel & -0.45 & -0.17 & 0.15 & 0.50 & 0.32 & 0.95 \\
\hline parathion & -0.56 & -0.30 & 0.26 & 0.60 & 0.57 & 1.16 \\
\hline pentachlorophenol & -0.38 & -0.17 & 0.13 & 0.40 & 0.31 & 0.78 \\
\hline zinc & -0.20 & -0.12 & 0.06 & 0.25 & 0.18 & 0.46 \\
\hline
\end{tabular}

\section{Results and discussion}

Duboudin et al [4] have combined in a near-comprehensive experimental design the different above-indicated options to handle ecotoxicological data, yielding, from the same set of data 63 distinct ways of calculating an HC5 (different statistical methods to build SSDs were also included in their analysis - not presented in this paper). This experimental design was tested on 15 toxicants, using chronic aquatic NOEC (No Observed Effect Concentration) data available for these toxicants. A comparison of these various approaches was then undertaken, with a view to determining the variability of the HC5 according to the chosen assumptions and to determining the respective effect of each of the options on the calculation of the HC5.

Results are summarized in Table 1. The deviation to the mean and the amplitudes of the outputs (distances max-min and $75^{\text {th }}$ percentile- $25^{\text {th }}$ percentile, in $\log$ base 10) are indicated to show the variability of the HC5 according to the 
assumptions chosen to handle data. The 'max-min' amplitude of the HCs5 calculated according to the various assumptions varies from one toxicant to another: it lies between 0.3 (in log base 10) for butylbenzyl phthalate and 1.19 (i.e. more than one order of magnitude for concentrations) for boric acid; it exceeds 0.5 (factor of 3 ) in 12 cases out of 15 . An ANOVA analysis conducted for each toxicant showed that: (i) the parameter related to 'taxonomic distribution of data' turns out to be the preponderant parameter for variations in HC5 for 7 toxicants out of 15; (ii) the 'intra-species variation' parameter is preponderant for 5 toxicants out of 15. Thus, the ranking in order of importance of the studies parameters varies according to the toxicant and the related set of data.

\subsubsection{Recommendations}

The study conducted by Duboudin et al [4] showed that different methods can be separated by one order of magnitude for the same toxicant. Regardless of differences in toxicity between substances, the HC5s differ by the options used, firstly in terms of taxonomic groups and intra-species variation, and to a lesser degree in terms of the choice of distribution. In order to obtain comparable HC5 between substances, it could be recommended to use a homogeneous method, that allows one to take into account intra-species variations and constant distribution of data among taxonomic groups:

- $\quad$ regarding redundant data for each species, rather that using a geometric mean as done by the current method, we favor a weighted method that allows one to account for intra species variation while giving the same weight within the SSD.

- $\quad$ regarding the proportion of data among taxonomic groups, we favor weighted methods that allow one to conduct a homogeneous treatment for all the toxicants.

\subsection{Strategies for large acute datasets}

\subsubsection{Materials and methods}

For many substances, insufficient or even no chronic data are available. In such cases, the PNEC is based on a limited number of data and on uncertainty factors $(10,100,1000$ depending on the case [1]): only the lowest value is used to estimate the PNEC and no confidence interval can be allotted to it. However, for some of these substances, a large number of acute data may be available. Then, we are faced with the following problem: (question 3.1.) can acute toxicity data for a substance give us information about the chronic toxicity of the same substance and be used to build chronic SSDs?

Some methods were developed in the past, that are based on the transformation of type $\mathrm{LC}_{50}$ or $\mathrm{EC}_{50}$ acute toxicity data concerning one species into predicted chronic data for the same species, using an ACR (Acute to Chronic Ratio) (e.g. Parkurst et al [5]). However, the ACR method showed some limitations: significant differences in ACR ratios among different families of toxicants or species (e.g. Länge et al [6]; Brix et al [7]). So in order to apply this 
approach correctly it would be necessary to know the ACR ratios for each family of substances, and virtually for every species, which is impossible.

Duboudin et al [8] compared, for several substances, SSDs constructed from acute toxicity data with SSDs constructed from chronic data, and more specifically the mean and standard statistics of the log-normal distributions associated with both of them. They developed thus an Acute-to-Chronic Transformation method allowing one to incorporate acute data in the construction of chronic SSDs.

Table 2: Inter-distribution distances HC5s for Acute, Chronic and ACT SSDs, for 11 substances (data in log base 10). ACT = acute to chronic data transformation; $\mathrm{A}=$ acute and $\mathrm{C}=$ chronic.

\begin{tabular}{|l|c|c|c|c|c|}
\hline Substances & \multicolumn{2}{|c|}{$\begin{array}{l}\text { Interdistribution } \\
\text { distance }\end{array}$} & \multicolumn{3}{|c|}{ HC5 } \\
\hline & $\mathrm{A}-\mathrm{C}$ & $\mathrm{ACT}-\mathrm{C}$ & $\mathrm{A}$ & $\mathrm{ACT}$ & $\mathrm{C}$ \\
\hline cadmium & 2.39 & 0.14 & 0.69 & -0.23 & -0.29 \\
\hline copper & 1.23 & 0.06 & 0.99 & 0.22 & 0.22 \\
\hline nickel & 1.89 & 0.12 & 2.55 & 0.87 & 0.80 \\
\hline lead & 1.60 & 0.18 & 1.71 & 0.68 & 1.09 \\
\hline zinc & 1.53 & 0.06 & 2.18 & 1.08 & 1.22 \\
\hline mercury & 1.27 & 0.27 & 0.86 & 0.11 & -0.53 \\
\hline chromium & 1.67 & 0.16 & 2.09 & 0.93 & 0.97 \\
\hline lindane & 0.49 & 0.02 & 0.74 & -0.10 & -0.11 \\
\hline atrazine & 2.05 & 0.34 & 3.34 & 0.89 & 0.71 \\
\hline parathion & 1.28 & 0.16 & -0.31 & -1.20 & -1.58 \\
\hline pentachloro- & 0.69 & 0.26 & 1.36 & 0.48 & 0.90 \\
phenol & & & & & 0.31 \\
\hline Mean & 1.46 & 0.16 & 1.47 & 0.34 & \\
\hline
\end{tabular}

${ }^{1}$ defined as $\mathrm{D}\left(\mathrm{d}_{1}, \mathrm{~d}_{2}\right)=\lim _{\mathrm{n} \rightarrow \infty}\left[\frac{1}{\mathrm{n}} \sum_{\mathrm{k}=0}^{\mathrm{n}-1} \mid\right.$ quantile $_{\mathrm{k} / \mathrm{n}-1}\left(\mathrm{~d}_{1}\right)-$ quantile $\left._{\mathrm{k} / \mathrm{n}-1}\left(\mathrm{~d}_{2}\right) \mid\right]$

\subsubsection{Results and discussion}

Duboudin et al [8] developed relationships to transform a data sample representative of the acute toxicity of a substance on the vertebrates and invertebrates into a data sample predicted to be representative of the chronic toxicity of the same substance (ACT method). This method was tested and validated on 11 substances for which a sufficient number of chronic and acute data are available. For each of these substances, three SSDs were constructed and compared using different sets of data: (i) SSD with acute data only; (ii) SSD with chronic data only; (iii) predicted chronic SSD using acute-to-chronic transformation data (ACT SSD). Results of HC5s obtained with these methods 
are summarized in Table 2: the inter-distributions mean distance is 1.46 between acute and chronic data as opposed to 0.16 between ACT and chronic; for the 11 substances under consideration, while the difference in absolute values (Table 6) between acute $\mathrm{HC} 5 \%$ and chronic $\mathrm{HC} 5 \%$ is on average 1.16 in log base 10, it is 0.21 on average between ACT $\mathrm{HC} 5 \%$ and chronic $\mathrm{HC} 5 \%$, which represents a ratio in the region of 1.6 between concentration values. These results confirm that the ACT method is successful at reproducing a distribution of chronic toxicity data from samples of acute data and predicting a chronic $\mathrm{HC} 5 \%$ value, even if the quality of the prediction varies from one substance to another.

\subsubsection{Recommendations}

The ACT approach developed by Duboudin et al [8] thus has two main advantages over the assessment factors method: first, rather than providing a single value, it gives a distribution of vertebrate and/or invertebrate data, associated with a confidence envelope for acute-to-chronic transformation. This or these distribution(s) of predicted chronic data can be compared with real available chronic values if existent. The few available chronic values can be incorporated into the acute values after ACT transformation and thus be taken into account when constructing an SSD. Second, if completed successfully (incorporation of the three taxonomic groups into the same SSD with the wished representativeness for each one), the ACT approach provides an $\mathrm{HC} 5 \%$ value associated with a confidence interval which takes into account, on the one hand, natural variation of data, and on the other, the uncertainty of the acute-to-chronic transformation.

\subsection{Strategies for small datasets}

For most of the toxicants, available data are scarce and, in such cases, the assessment method is applied: as previously indicated, uncertainty factors $(10$, 100,1000 depending on the case [1]) are affected to the lowest ecotoxicological value to estimate the PNEC and no confidence interval can be allotted to it. In such cases, different alternative methods can be proposed to build SSDs, even with a limited set of data (see Verdonck et al [9]). Among these methods, Bayesian statistics corresponds to practical situation that end-users meet: a limited data sample is available and the uncertainty of the SSD that fits the dataset is modelled assuming that the parameters of the SSD are themselves distributed.

Aldenberg and Jaworska [10] used theoretical sets of data to compare 'classical' statistics (i.e. maximum likelihood method) and Bayesian methods to derive HC5. They provided tabulations for direct applications of Bayesian methods for the calculation of $\mathrm{HC} 5 \mathrm{~s}$, associated with confidence intervals, as a function of the number of available tests. An extract of the table provided by Aldenberg and Jaworska [10] is proposed in Table 3. The methods allows thus to quantify the confidence interval of the HC5, that decreases when the number of data increases. Moreover, it is observed that the generation of new ecotoxicological data (e.g. from 5 to 7 data) allows reducing the conservatism of the predicted PNEC. This method has thus main advantages over the assessment 
factors method: it uses all the available ecotoxicological data (and not the lowest one as for the assessment factor method); it provides a confidence interval for HC5; it stimulates the generation of new data that can contribute to limit the conservatism of the predictions.

Table 3: Calculation of HC5s by the Bayesian method as a function of sample size (from Aldenberg and Jaworska [18]).

\begin{tabular}{|l|c|c|c|}
\hline & \multicolumn{3}{|c|}{$\mathrm{HC}=$ mean $-k$.standard_deviation } \\
\hline Number of data & $k$ for HC5 $(5 \%)$ & $k$ for HC5 $(50 \%)$ & $k$ for HC5(95\%) \\
\hline 5 & 4.2 & 1.78 & 0.82 \\
\hline 7 & 3.4 & 1.73 & 0.92 \\
\hline 10 & 2.91 & 1.7 & 0.99 \\
\hline infinity & 1.64 & 1.64 & 1.64 \\
\hline
\end{tabular}

\section{Conclusions}

This paper summarizes previous works undertaken to improve the determination of PNEC values, using preferentially the Species Sensitivity Distribution (SSD) method. It underlines some possible strategies for calculating HC5s according to the size of data sample:

- when the number of chronic data is sufficient (e.g. more than 15 data), it could be recommended to use a homogeneous method, that allows to take into account intra-species variations and constant distribution of data among taxonomic groups;

- when the chronic dataset is limited, but acute dataset is sufficient, an Acute-to-Chronic Transformation method could be used to incorporate acute data in the construction of the chronic SSD;

- when only a few number of data is available, Bayesian techniques (already tabulated) could be used to build SSDs, with associated confidence intervals.

\section{References}

[1] European Commission. 1996. Technical Guidance Document in support of commission directive 93/67/EEC on risk assessment for new notified substance and commission regulation 5EC) No 1488/94 on risk assessment for existing substance. Part II. Office for Official Publications of the European Communities. Luxembourg. CR-48-96-001-EN-C ; CR48-96-002-EN-C ; CR-48-96-003-EN-C ; CR-48-96-004-EN-C.

[2] Aldenberg T, and Slob W. 1993. Confidence limits for hazardous concentrations based on chronic NOEC toxicity data. Ecotoxicology and Environmental Safety 25:48-63.

[3] Forbes VE and Calow P. 2002. Species Sensitivity Distributions Revisited: A critical Appraisal. Human an Ecological Risk Assessment $8: 3: 473-492$. 
[4] Duboudin C, Ciffroy P, Magaud H, 2004. Effects of data manipulation and statistical methods on species sensitivity distribution. Environmental Toxicology and Chemistry, Vol. 23, No 2, 489-499.

[5] Parkurst BR, Warren-Hicks W, Cardwell RD, Volosin J, Etchison T, Butscher JB, Covington SM. 1995. Risk managing methods. Water Environ Technol 7:39-43

[6] Länge R, Hutchinson TH, Scholz N, Solbe J. 1999. Analysis of the Ecetoc aquatic toxicity (EAT) database. II - Comparison of acute to chronic ratios for various aquatic organisms and chemical substances. Chemosphere $36: 1: 115-127$.

[7] Brix KV, Deforest DK, Adams WJ. 2001. Assessing acute and chronic copper risks to freshwater aquatic life using species sensitivity distributions for different taxonomix groups. Environ Toxicol Chem 20:8:1847-1856.

[8] Duboudin C, Ciffroy P, Magaud H, 2004. Acute-to-chronic species sensitivity distribution extrapolation. Environmental Toxicology and Chemistry, Vol. 23, No 7, 1774-1785

[9] Verdonck F, Jaworska J, Thas O, Vanrolleghem P., 2001. Determining environmental standards using bootstrapping, Bayesian and maximum likelihood techniques: a comparative study. Analytica Chimica Acta, Vol 446, Issues 1-2, 427-436

[10] Aldenberg T, Jaworska J, 2000. Uncertainty of the hazardous concentration and fraction affected for normal Species Sensitivity Distribution. Ecotoxicology and Environmental Safety, 46, 1-18. 